\title{
Gel-Permeation Chromatography and Intrinsic Viscosity of Poly(4-vinylbenzyltrimethylammonium chloride) in Aqueous Solution
}

\author{
Yuji Higo, * Yoshio Kato, Masaki Itoh, Norio KozUKA, \\ Ichiro NoDA, and Mitsuru NAGASAWA \\ Department of Synthetic Chemistry, Nagoya University, \\ Furo-cho, Chikusa-ku, Nagoya 464, Japan
}

(Received May 6, 1982)

\begin{abstract}
Gel permeation chromatography and viscosity behavior of a cationic polyelectrolyte, poly(4-vinylbenzyltrimethylammonium chloride), in aqueous solution were studied as an appropriate eluting solvent for GPC, $0.1 \mathrm{~mol}^{-1}$ sodium sulfate containing $2.5 \%$ acetic acid was used with this eluting solvent, the molecular weight distribution of this polycation could be determined with no difficulty. It was found that the intrinsic viscosity of a chloride salt of this polycation as a function of molecular weight and added salt concentration followed the relation presented for poly(sodium acrylate) in aqueous sodium chloride. Moreover, from the difference in viscosity behavior between the chloride and iodide salts of the polycation the existence of a specific interaction between iodide ion and the trimethylammonium group was suspected.
\end{abstract}

KEY WORDS Poly(4-vinylbenzyltrimethylammonium chloride) / Polyelectrolyte / GPC / Intrinsic Viscosity /

In a previous paper ${ }^{1}$ we reported the preparation of a cationic polyelectrolyte, poly(4-vinylbenzyltrimethylammonium chloride) (P4VBTMA-Cl), having relatively narrow molecular weight distributions by anionic polymerization technique. This polymer sample is useful since few polycations having narrow molecular weight distributions are available. At present, however, it is not easy to prepare samples very high in molecular weight and narrow in molecular weight distribution by this method, because of the difficulty in purifying the monomer. ${ }^{1}$ To improve the preparation method it is necessary to establish a characterization method for the sample.

One purpose of this work has been to study the gel permeation chromatography (GPC) of P4VBTMA-Cl. GPC has been extensively used for characterizing non-ionic polymers in organic solvents. However, it is only recently that this technique has been applied to water soluble polymers and in particular, to polyelectrolytes. ${ }^{2-11}$ Moreover, various difficulties may be found in GPC if the sample is a polycation. In this study an attempt was also made to compare the viscosity behavior of this polycation with that of poly(sodium acrylate). Since very abnormal viscosity behavior is often found in working with polycations, ${ }^{12,14}$ it was considered worthwhile to accumulate viscosity data on well-characterized polycation samples.

\section{EXPERIMENTAL}

\section{Samples}

Poly(4-vinylbenzyldimethylamine) (P4VBDMA) was prepared and quaternized to poly(4-vinylbenzyltrimethyl ammonium chloride and iodide) (P4VBTMA-Cl and -I) by the same procedure reported previously. ${ }^{1}$ The degree of quternization was nearly $100 \%$ for all samples. The number-average molecular weights $\left(M_{n}\right)$ of P4VBDMA were determined in toluene at $25^{\circ} \mathrm{C}$ using a Hewlett-Pakard high speed membrane osmometer, (type 502). The $M_{n}$ of P4VBTMA-Cl was evaluated from that of

* Address: Central Research Laboratory, Toyo Soda Mfg. Co., Ltd., Tonda, Shinnanyo, Yamaguchi 746, Japan. 
P4VBDMA, assuming that the degree of polymerization undergoes no change in quternization. ${ }^{1}$ The $M_{n}$ of the samples thus evaluated are listed in Table I. Sample TH8M had a rather broad molecular weight distribution, while those of other four samples were probably rather narrow.

Acetic acid, sodium sulfate and acetonitrile used for GPC measurements and also sodium chloride used as added salt for the viscosity measurements were of GR grade.

\section{GPC Measurements}

GPC measurements were carried out at $25^{\circ} \mathrm{C}$ with a Toyo Soda liquid chromatograph HLC-803C, using TSK-GEL PW type columns (G3000PW and $\mathrm{G} 5000 \mathrm{PW}$ ) commercially available for use in aqueous solutions. The flow-rate was $0.7 \mathrm{ml} \mathrm{min}^{-1}$, the polymer concentration was $2 \mathrm{mg} \mathrm{ml}^{-1}$, and the sample volume was $100 \mu$ l. The standard polymers used for the calibration of the GPC columns were TSK standard poly(ethylene oxide)s (PEO) of Toyo Soda with weight-average molecular weights of $2.8 \times 10^{3}-27.8 \times 10^{4}$.

\section{Viscosity Measurements}

Viscosity measurements were carried out using a modified Ubbehlode viscometer with a negligible kinetic energy correction. The shear-rate dependence was negligible. The intrinsic viscosity $[\eta]$ was determined as the common intercept of the plots of $\eta_{\mathrm{sp}} / c v s . c$ and $\ln \left(\eta_{\mathrm{r}}\right) / c$ vs. $c .^{14,15}$

\section{RESULTS AND DISCUSSION}

\section{$G P C$ and $M_{w} / M_{n}$}

One problem related to the GPC of polyelectrolytes is that the radius of gyration of a polyelectrolyte changes so markedly with an increase in salt concentration that it is difficult to establish the relationship between the elution volume and molecular weight. Another problem is the interaction between polyelectrolytes and the gel matrix. For example, the matrix of the PW type gels of Toyo Soda has a tendency to adsorb polymers having cationic, hydrophobic, or in particular aromatic groups. ${ }^{8}$ The adsorption is probably caused by small amounts of carboxyl and hydrophobic groups in the gel matrix. ${ }^{2,8}$ It is known that the hydrophobic interaction between polyelectrolytes and the gel matrix may be diminished by adding a polar organic reagent such as acetonitrile to eluting solvent, and also that the interaction between polycations and carboxyl groups of the gel matrix may be diminished by the use of an acidic eluting solvent. ${ }^{8,9}$

On the basis of above considerations the GPC of TH8M was measured to find an eluting solvent suitable for our polyelectrolyte. As shown in Figures $1 \mathrm{a}$ and $1 \mathrm{~b}$, the elution curves in $0.1 \mathrm{~mol}^{-1}$ phosphate buffer $(\mathrm{pH}=2.0)$ and $0.1 \mathrm{~mol}^{-1}$ sodium sulfate, commonly used for GPC of aqueous solutions, showed an unusual discontinuous rise on the high molecular weight side. Since the column was made of stainless steel which corrods easily with halogen ions in acidic solutions, aqueous solutions of halides such as sodium chloride were not tested. The addition of $5-10 \%$ acetonitrile was not effective as can be seen from Figures $1 \mathrm{a}$ and $1 \mathrm{~b}$. However, as shown in Figure 1c, normal GPC elution curves, were obtained when $0.1 \mathrm{~mol}^{-1}$ sodium sulfate solution containing small amounts of acetic acid were used as eluents.

The GPC of four P4VBTMA-Cl samples in this eluting solvent are shown in Figure 2. These curves are not so narrow that the elution volumes of their maxima do not correspond to the number-average molecular weights $M_{n}$ of the samples. Moreover, it

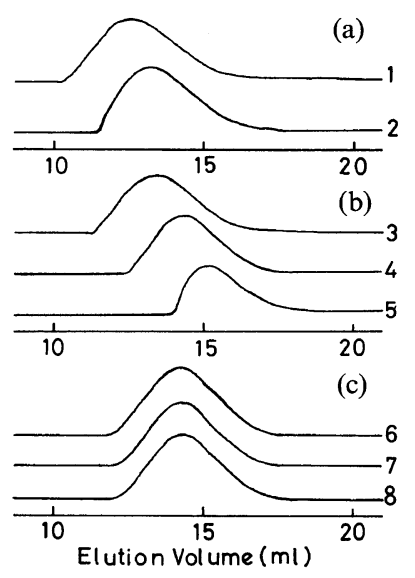

Figure 1. GPC elution curves of P4VBTMA-Cl in various eluting solvents: (a) $1,0.1 \mathrm{~mol}^{-1}$ phosphate buffer $+10 \% \mathrm{CH}_{3} \mathrm{CN} ; 2,0.1 \mathrm{~mol} \mathrm{l}^{-1}$ phosphate buffer. (b) 3, $0.1 \mathrm{moll}^{-1} \mathrm{Na}_{2} \mathrm{SO}_{4}+10 \% \mathrm{CH}_{3} \mathrm{CN} ; 4,0.1 \mathrm{~mol} \mathrm{l}^{-1}$ $\mathrm{Na}_{2} \mathrm{SO}_{4}+5 \% \mathrm{CH}_{3} \mathrm{CN} ; 5,0.1 \mathrm{~mol} \mathrm{l}^{-1} \mathrm{Na}_{2} \mathrm{SO}_{4}$. (c) 6, 0.1 mol $1^{-1} \quad \mathrm{Na}_{2} \mathrm{SO}_{4}+5 \% \mathrm{CH}_{3} \mathrm{COOH} ; 7,0.1 \mathrm{~mol} \mathrm{l}^{-1}$ $\mathrm{Na}_{2} \mathrm{SO}_{4}+2.5 \% \mathrm{CH}_{3} \mathrm{COOH} ; 8,0.1 \mathrm{~mol} \mathrm{l}^{-1} \mathrm{Na}_{2} \mathrm{SO}_{4}$ $+1 \% \mathrm{CH}_{3} \mathrm{COOH}$. 


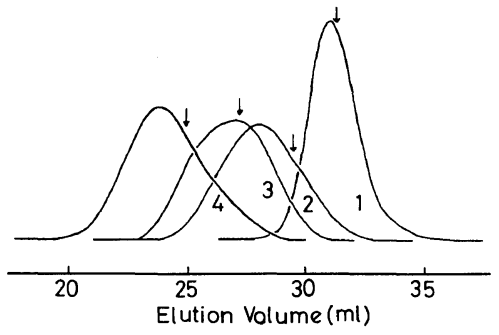

Figure 2. GPC elution curves of P4VBTMA-Cl: 1, TH11M; 2, TH17M; 3, TH10M; 4, TH12M. The elution volumes indicated by arrows correspond to $M_{n}$ of the samples.

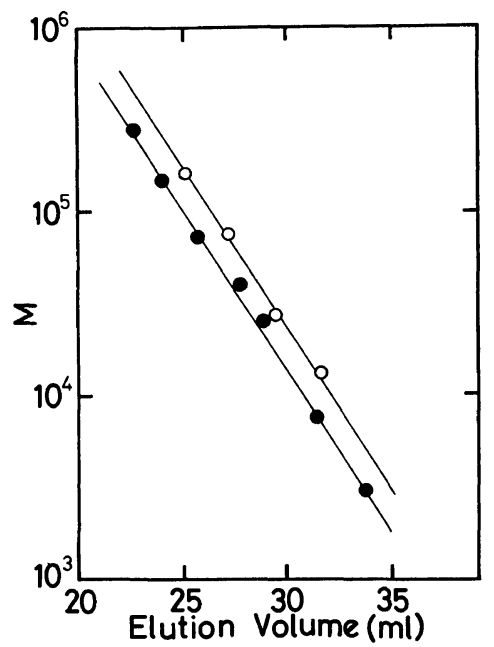

Figure 3. Relations between $M_{n}$ and $V_{\mathrm{e}}$. P4VBTMA$\mathrm{Cl}(\mathrm{O})$, PEO (O).

was recently reported that the universal calibration curve does not always hold for polyelectrolytes. ${ }^{11}$ In this work, therefore, we assumed a linear relationship between elution volume $V_{\mathrm{e}}$ and $M_{n}$, and modified by trial and error the linear relationship in such a way that $M_{n}$ determined by osmometry and those calculated from chromatograms using the relationship are in agreement. Figure 3 shows the $V_{\mathrm{e}}-M_{n}$ relationship thus determined. The values of $V_{\mathrm{e}}$ corresponding to $M_{n}$ of the samples are shown by arrows in Figure 2.

The $V_{\mathrm{e}}-M_{n}$ relationship for the standard samples (PEO) in the same eluting solvent was also determined. The result is shown in Figure 3. In this case, the calibration curve was constructed by plotting the maximum elution volumes against the $M_{w}$ of the standard samples, since the molecular weight

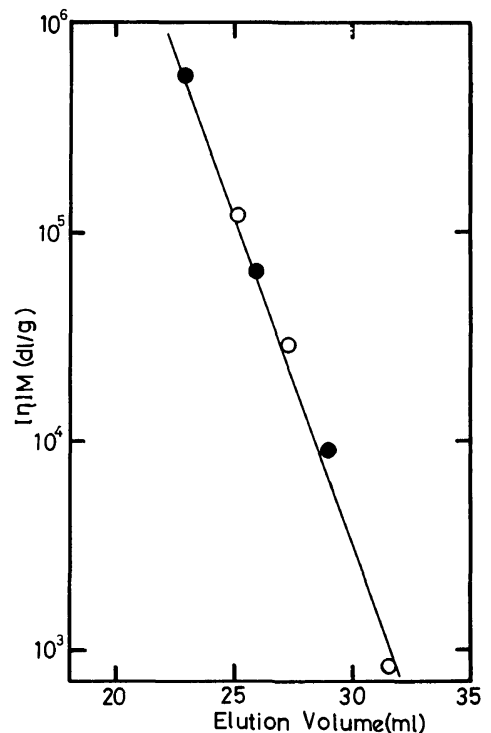

Figure 4. Universal calibration curves. P4VBTMA-Cl (O), PEO (O).

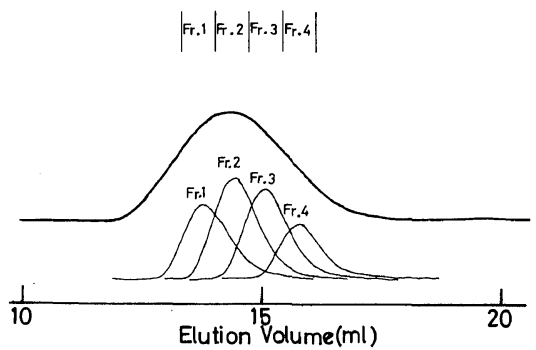

Figure 5. GPC elution curves of sample TH8M and its fractions.

distributions of samples used were narrow.

The values of $[\eta] M_{n}$ of both P4VBTMA-Cl and PEO, which were calculated from the data in Figure 3 and the experimental values of $[\eta]$, are plotted against the elution volumes in Figure 4. It can be seen that a common relationship hold for both P4VBTMA-Cl and PEO. This fact indicates that the molecular weight distributions of P4VBTMA-Cl may be determined from their gel permeation chromatographs using the universal calibration curve constructed with PEO.

In order to confirm the suitability of a $0.1 \mathrm{~mol}^{-1}$ sodium sulfate solution containing $2.5 \%$ acetic acid as an eluting solvent for the present system, we took four fractions of sample TH8M near the peak of 
chromatogram and measured the GPC elution curve of each fraction. The elution volume ranges of these four fractions and the chromatogram of each fraction are shown, along with the chromatogram of the parent sample TH8M, in Figure 5. This figure indicates that fractionation of the parent sample TH8M may be carried out with respect to molecular weight without any abnormality.

The reason for the suitability of $0.1 \mathrm{~mol} \mathrm{l}^{-1}$ sodium sulfate solution containing $2.5 \%$ acetic acid may be that the dissociation of carboxyl groups of the gel is suppressed in acidic solutions so that the electrostatic adsorption of polycation on the gel matrix is negligible, and also that the hydrophobic interaction between the solute and the gel matrix is diminished by the addition of acetic acid. ${ }^{9,10}$ As is well known, the addition of acetic acid is effective in removing the specific interaction between the solute and the gel matrix when the solute contains aromatic groups. ${ }^{9}$ Moreover, no specific interaction appears to exist between the electrolytes in this eluting solvent and our polycation, since the viscosity behavior of the polycation in this eluting solvent is normal.

The ratios of weight-average molecular weight to number-average molecular weight $\left(M_{w} / M_{n}\right)$ of the present samples can be evaluated from GPC using the $V_{\mathrm{e}}-M_{n}$ relation in Figure 3. The values of $M_{w} /$ $M_{n}$ thus determined are listed in Table I. GPC chromatograms are generally broadened by the axial dispersion of solute in the columns. Although various correction methods have been proposed for this effect, in this work, we applied none of these to the chromatograms of our samples. Table I shows that the molecular weight distribution of P4VBTMA-Cl is broader for a higher molecular weight sample. This agrees with our prediction from the study of polymerization kinetics. ${ }^{1}$

\section{Viscosity Behavior of P4VBTMA-Cl}

Figure 6 shows double logarithmic plots of $(\eta) v s$. $M_{n}$ for P4VBTMA-Cl in sodium chloride solutions at various ionic strengths at $25^{\circ} \mathrm{C}$. The constant $v$ and $K$ in the viscosity equation $[\eta]=K M_{n}{ }^{v}$ are listed in the figure caption. The values were found to depend strongly on ionic strength as is usually the case for polyelectrolytes. For low molecular weight samples of poly(sodium acrylate) at various degrees of neutralization, the following relation was found to hold among intrinsic viscosity $[\eta]$, concentration
Table I. Molecular characteristics of P4VBTMA-Cl

\begin{tabular}{ccc}
\hline Sample code & $M_{n} \times 10^{-4}$ & $M_{w} / M_{n}$ \\
\hline TH11M & 1.3 & 1.07 \\
TH17M & 3.8 & 1.67 \\
TH10M & 7.8 & 1.40 \\
TH12M & 16.8 & 1.47 \\
TH8M & 21.0 & - \\
\hline
\end{tabular}

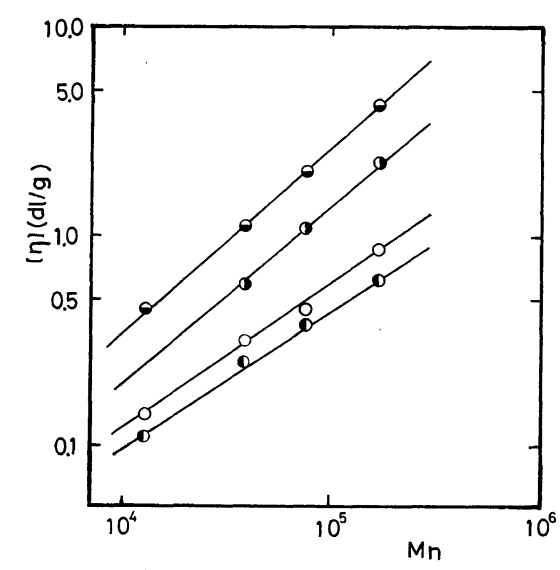

Figure 6. The constants in the Mark-HowinkSakurada equation for P4VBTMA-Cl.

\begin{tabular}{|c|c|c|c|}
\hline \multirow{2}{*}{ Symbol } & $C_{\mathrm{s}}$ & \multirow{2}{*}{$K \times 10^{4}$} & \multirow{2}{*}{$v$} \\
\hline & $\mathrm{mol} \mathrm{l}^{-1}$ & & \\
\hline D & 0.5 & $1.9_{6}$ & 0.67 \\
\hline 0 & 0.1 & $1.8_{6}$ & 0.70 \\
\hline 1 & 0.01 & $0.78_{7}$ & 0.85 \\
\hline$\theta$ & 0.002 & $0.57_{7}$ & 0.88 \\
\hline
\end{tabular}

of sodium chloride $C_{\mathrm{s}}$, and molecular weight $M \cdot^{15,16}$

$$
[\eta] / M^{1 / 2}=K_{0}+0.5 \Phi_{0} B\left(M / C_{\mathrm{s}}\right)^{1 / 2}
$$

where $\Phi_{0}$ is the Flory viscosity constant, $B$, the thermodynamic interaction parameter, and $K_{0}$ the value of $K$ at the $\theta$-state. In Figure 7, the intrinsic viscosities of P4VBTMA-Cl in sodium chrolide solutions are plotted according to eq 1. A linear relation can be seen as in the case of the sodium salts of poly(acrylic acid). The intercept $K_{0}$ is related to unperturbed square end-to-end distance $\left\langle R^{2}\right\rangle_{0}$ by $K_{0}=\Phi_{0} \quad\left(\left\langle R^{2}\right\rangle_{0} / M\right)^{3 / 2}$. If we assume $\Phi_{0}=$ $2.87 \times 10^{21}$, we have $4.09 \times 10^{-17}\left(\mathrm{~cm}^{2} \mathrm{~g}^{-1}\right)$ for $\left\langle R^{2}\right\rangle_{0} / M$ of P4VBTMA-Cl, and then 3.2 for the 


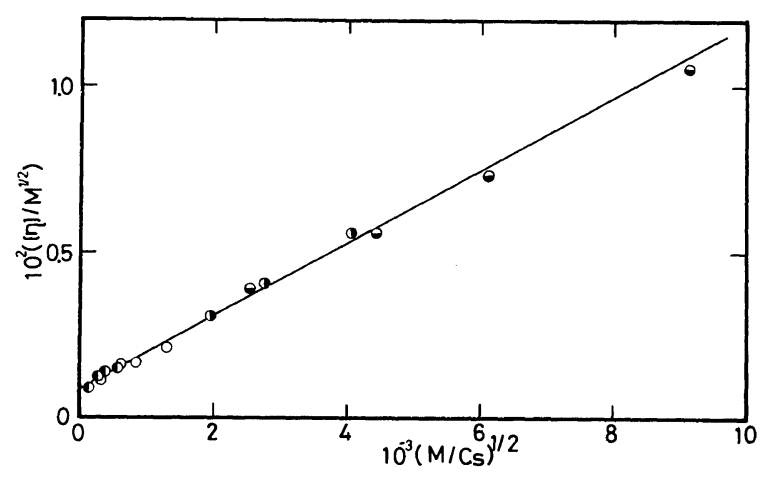

Figure 7. $[\eta] / M^{1 / 2}$ vs. $\left(M / C_{\mathrm{s}}\right)^{1 / 2}$ plot for P4VBTMA- Cl. The symbols are the same as in Figure 6.

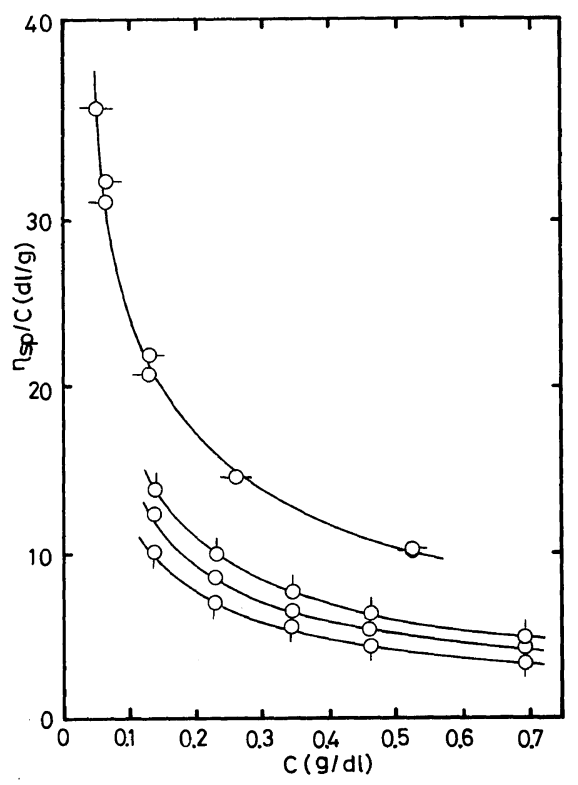

Figure 8. Polymer concentration dependence of specific viscosities of P4VBTMA-Cl and -I at various temperatures: P4VBTMA-Cl, (-O) $5.8^{\circ} \mathrm{C} ;\left(\mathrm{O}^{-}\right) 25^{\circ} \mathrm{C}$; P4VBTMA-I, (O) $5^{\circ} \mathrm{C}$; (O) $15^{\circ} \mathrm{C}$; (O) $25^{\circ} \mathrm{C}$.

characteristic ratio $\sigma .^{17}$ This value of $\sigma$ is somewhat larger than those of poly(styrene) (2.3) and poly(sodium acrylate) (2.4). ${ }^{17}$ This difference is understandable, considering that P4VBTMA-Cl has a large substituent group, benzyltrimethylammonium.

Finally we discuss briefly the effect of counter ion species on specific viscosity of our polyelectrolyte in the absence of added salt. Figure 8 shows the concentration dependence of specific viscosities of
P4VBTMA-Cl and -I (sample TH8M) in pure water at various temperatures. A considerable difference can be seen in the viscosity behavior of P4VBTMA$\mathrm{Cl}$ and $-\mathrm{I}$; the specific viscosity of P4VBTMA-I is much smaller than that of P4VBTMA-Cl, and the former strongly depends on temperature whereas the latter does not. Moreover, it was found that a $5 \%$ aqueous solution of P4VBTMA-I became turbid even in the absence of added salt when the temperature was lowered to $25^{\circ} \mathrm{C}$. These facts imply that there may be a specific interaction between the polycation and the iodide ion in addition to the electrostatic interaction. However, no such specific interaction need be considered for the chloride salt, in agreement with the conclusion ${ }^{12}$ derived from a previous study an another polycation poly(vinylbenzyltrimethylammonium salt).

\section{REFERENCES}

1. Y. Higo, H. Chosi, T. Fujimoto, and M. Nagasawa, Polym. J., 12, 729 (1980).

2. T. Hashimoto, H. Sasaki, M. Aimura, and Y. Kato, J. Polym. Sci., Polym. Phys. Ed., 16, 1789 (1978).

3. A. Domard, M. Rinaudo, and C. Rochas, J. Polym. Sci., Polym. Phys. Ed., 17, 678 (1978).

4. C. Rochas, A. Domard, and M. Rinaudo, Eur. Polym. J., 16, 135 (1980).

5. C. Rochas, A. Domard, and M. Rinaudo, Polymer, 26, 76 (1979).

6. W. Siebourg, R. D. Lundberg, and R. W. Lenz, Macromolecules, 13, 1013 (1980).

7. J. E. Rollings, A. Bose, J. M. Caruthers, M. R. Okos, and G. T. Tsao, Polym. Prepr., Am. Chem. Soc., Div. Polym. Chem., 22, 294 (1981).

8. Y. Kato and T. Hashimoto, J. Chromatogr., 235, 589 (1982). 
9. J. C. Janson, J. Chromatogr., 28, 121 (1967).

10. D. Eaker and J. Porath, Separation Sci., 2, 507 (1967).

11. T. Kato and A. Takahashi, Polym. Prepr., Jpn., 30, 1764 (1981).

12. I. Noda and I. Kagawa, Kogyo Kagaku Zasshi, 67, 1423 (1964).

13. U. P. Strauss, N. S. Gersheld, and H. Spiera, J. Am. Chem. Soc., 76, 5909 (1954).
14. A. Takahashi and M. Nagasawa, J. Am. Chem. Soc., 86, 543 (1964).

15. I. Noda, T. Tsuge, and M. Nagasawa, J. Phys. Chem., 74, 710 (1970).

16. W. H. Stockmayer and M. Fixman, J. Polym. Sci., C, 1, 137 (1963).

17. J. Brandrup and E. H. Immergut, Ed., "Polymer Handbook," 2nd ed, Wiley-Interscience, New York, N.Y., 1975. 